of journals will continue. Neither potential readers nor actual libraries are multiplying at anything like the rate that journals have increased in number in the last decade. The rising costs of production are now forcing libraries to be more selective, and librarians are assessing the quality of journals and the amount they are used.

Professor Henry Harris made some useful comments on the features that maintain quality in scientific journals. The scope of the journal-in both subject matter and potential authors-had to be large enough to ensure that enough papers were submitted for the poor ones to be rejected. The referees should be young and energetic rather than old, distinguished, and too busy; and the editors should know enough about the subject to recognize capricious refereeing. Perhaps, as one speaker suggested, referees' names should be printed alongside the papers they recommended. Professor Harris was also unimpressed by the emphasis given to speed of publication by some journals. "If there is that much of a scramble for priority," he observed, "then the work can't have any real originality."

\section{Torsion of the Testicle Again}

Earlier this year a paper by R. H. Chapman and A. J. Walton $^{1}$ and a leading article ${ }^{2}$ drew attention to torsion of the testicle as a remediable condition often missed by doctors who would be ashamed to miss an acute appendix or a perforated peptic ulcer. The ensuing correspondence showed the persistence of the old notion that torsion is rare or discounted the existence of the underlying anomaly in the construction of the tunica vaginalis which permits the testicle to spin round on its mesentery. Acute torsion is sometimes missed, sometimes treated as if it were epididymitis, and sometimes not offered the chance of manipulative reduction, which in the early stages may save the testicle.

Attacks of pain in the lower abdomen ${ }^{3}$ or testicle and swelling in the testicle, the warning episodes which so frequently precede the last twist, are apt to be disregarded, 4 though if these warnings are heeded many testicles can be saved. J. A. Burton ${ }^{5}$ has recently drawn attention to the problem from the experience of the Sheffield Student Health Service, in which as medical officer he saw 17 examples of torsion within the short span of $3 \frac{1}{2}$ years. Unfortunately six of these were too late for anything to be done: the men had an atrophied testicle on one side. But in the same space of time he came across the same number in whom prompt manipulation followed by surgical fixation saved the gonad, thus confirming J. P. Sparks's similar experience at Rugby School. 6 Confronted with a possible example of testicular torsion, any doctor can make an attempt to untwist it there and then. In general the right testis should be "unscrewed" and the left one "screwed up" to undo the twist. If one forgets this in the heat of the moment, the simple mnemonic of "open the doors" may remind one which way the testicles should go. Or, if rotation of the testicle in one direction makes the pain worse, one may twist the other way. The manoeuvre is simple and worth trying.

Misconceptions about the rarity of torsion should be forgotten once and for all. M. Etienne and $\mathbf{M}$. Vialas ${ }^{7}$ in a recent review of the subject show that torsion may occur rather commonly and at any age. In the newborn boy it consists of extra-vaginalis torsion of the whole cord, and only this condition may be regarded as really uncommon. Later on in childhood it is the same kind of intra-vaginalis torsion as found in the adult, and unfortunately shares the same fate of being generally misdiagnosed and mismanaged. As in the adult, so in the infant, if there is any question of torsion, the testicle must be explored.

What is the risk of performing an unnecessary operation? Idiopathic oedema of the scrotum $^{8}$ may at first glance be confused with torsion, but the testicle itself feels normal in this strange condition; only the scrotal skin and fat are oedematous. Mumps orchitis is rare before puberty. Epididymitis does occur, but like epididymitis in the adult it usually begins by being limited to the epididymis and generally accompanies other evidence of urinary or urethral infection. If the testicle is undescended, there is less risk of the condition going unnoticed, since the tender lump will be diagnosed as a strangulated hernia and an operation performed without delay. The underlying anomaly which predisposes to torsion is often bilateral. Both testicles must be fixed if one has undergone torsion.

1 British Medical fournal, 1972, 1, 128.

2 Chapman, R. H., and Walton, A. J., British Medical fournal, 1972, 1, 164

3 Moore, T., British Medical fournal, 1972, 1, 374. and Epididymis. London, Butterworths, 1972.

s Burton, J. A., British fournal of Surgery, 1972, 59, 422.

- Sparks, J. P., Annals of the Royal College of Surgeons of England, 1971, 49, 77.

7 Etienne, M., and Vialas, M., Le Pediatre, 1972, 8, 9. Essenhigh, D. M., and Stewart, J. S. S., British fournal of Surgery, 1966,
53, 419.

\section{Candida Infection}

The unstable host-parasite relationship between man and Candida species, notably Candida albicans, is incompletely understood even by those who devote much of their time to the study of this increasingly impontant problem. Patients who are sympton-free healthy carriers of $C$. albicans in its non-pathogenic yeast phase frequently receive prolonged and unnecessary treatment. The development of acute candidiasis is favoured by certain antibiotics, corticosteroids, and perhaps by the contraceptive pill, and is now familiar to general practitioners.

The association of chronic candidiasis with diabetes mellitus has long been recognized, and the increased susceptibility to candidiasis of patients with some other endocrine disorders has also been known for many years. ${ }^{1}$ Chronic candidiasis may also occur in patients with a wide variety of defects in the immune response.

In $1970 \mathrm{R}$. S. Wells ${ }^{2}$ described a genetically determined syndrome in which susceptibility to chronic candidiasis occurs in the absence of other clinical abnormality. Wells and his colleagues ${ }^{3}$ have recently reported the results of their investigation of 46 patients, most of them referred at their request by dermatologists throughout England and Wales. All these patients had chronic oral candidiasis, some had candidiasis in other sites, but none had other clinically evident disease.

The 46 patients were provisionally classified in four groups. Twenty-two patients were placed in group 1; all had developed oral candidiasis early in life, often before the age of 2 , and some had candidiasis of nails or other sites. Pedigrees suggested that the susceptibility to candidiasis was probably determined by an autosomal recessive gene. Wells at present 